\title{
Relation of human cardiac action potential duration to the interval between beats: implications for the validity of rate corrected QT interval (QTc)
}

\author{
W A SEED, M I M NOBLE, P OLDERSHAW, R B WANLESS, \\ A J DRAKE-HOLLAND, D REDWOOD, S PUGH, C MILLS
}

From the Department of Medicine, Charing Cross and Westminster Hospital Medical School, London; King Edward VII Hospital, Midhurst, West Sussex; Department of Cardiology, Brompton Hospital, London; Department of Cardiology, Cardiothoracic Institute, London and Midhurst, West Sussex; and Departments of Medicine 1 and Cardiology, St George's Hospital Medical School, London

SUMMARY Fourteen patients undergoing routine cardiac catheterisation were paced at a stead产 frequency; after this test, stimuli were introduced with a variable preceding interval (test puls interval). The QT interval of the electrocardiogram and the duration of the monophasic action potential of the right ventricle were measured. QT interval is a function of action potentiat duration; the two variables were very closely correlated in this study. Both these variable increased in duration with increasing test pulse interval. A biphasic response, as previously reported, was not seen. An increase in steady state pacing frequency caused QT interval ane्ष action potential duration to decrease for any given $R-R$ interval. When frequency of stimulatio was suddenly increased and then maintained, there was an immediate action potential shortening followed by a further more gradual shortening occurring over several minutes. These results imply that a simple correction of QT interval for heart rate (QTc) is inadequate.

It is concluded that the relation between action potential duration (or QT interval) and hear rate depends on both the instantaneous interval between beats and the duration of the prevailing heart rate.

When the heart is electrically activated, depolarisation of the myocardium is registered as an upstroke of the action potential and as the QRS complex of the surface electrocardiogram. The period of myocardial depolarisation is indicated by the plateau of the action potential; this is terminated by repolarisation-that is the downstroke of the action potential and the $T$ wave of the electrocardiogram. The interval from the $Q R S$ to the $T$ wave of the electrocardiogram (QT interval) thus reflects the duration of the action potential. We have investigated the effect of heart rate on the QT interval using the more direct and accurate measurement of ventricular action potential duration. When an electrical impulse is delivered to the heart immedi-

Requests for reprints to Dr M I M Noble, King Edward VII Hospital, Midhurst, West Sussex GU29 0BL:

Accepted for publication 15 September 1986 ately after an action potential, the membrane is refractory and no response is obtained. When the stimulus is delivered a little later, action potentials are obtained which (in most species) are shorter tha those in the preceding steady state; they becoms longer as the interval between beats is increased. When this relation is explored formally by pacing at a given steady state frequency and by introducing $\$$ test pulse interval at progressively increasing intervals $^{12}$ a relation can be defined between actiofo potential duration (or QT interval) and test pulse interval ( $R-\mathbf{R}$ interval), which is termed the elece trical restitution curve. ${ }^{3-6}$ There is one study in man that included only five patients, two of whom showed prolongation of the action potential duration over a range of $R-R$ intervals of $300-400 \mathrm{~ms}^{6}{ }^{6}$ Th is unlike the findings in animals of Boyett and Jewell ${ }^{4}$ and Elzinga et al. ${ }^{5}$ The first objective in the present study was to examine electrical restitution ig 
a larger group of patients in order to establish the correct restitution curve.

Electrical restitution does not explain all the changes in action potential (and QT interval) that take place when the heart rate is increased. A sustained increase in heart rate causes a slow progressive shortening of QT interval and action potential duration over a period of several minutes. This has been shown for the guinea pig by Attwell $e t$ $a l^{7}$ and for intact dog by Drake et al. ${ }^{8}$ Attwell et al also showed that changes in human QT interval (which corresponds to the action potential duration) followed a similar pattern. ${ }^{7}$ The second objective of the present study was to examine the longer term response of action potential duration to an increase in heart rate. This is of considerable importance in understanding the relation of QT interval to cardiac frequency..$^{9-11}$ Bazett's correction of the QT interval (QTc) was formulated to allow for physiological variation in heart rate, assuming a linear relation. The results of Attwell et $\mathrm{al}^{7}$ strongly suggest that such correction is invalid and we therefore wished to confirm or deny that suggestion with a definitive study.

\section{Patients and methods}

\section{PATIENTS}

Fourteen patients were studied ( 9 men and 5 women, aged 36-74 years (table 1)). They were patients with ischaemic heart disease who were routinely undergoing cardiac catheterisation for diagnostic purposes. All drugs were stopped 24 hours before the study. No patients had received amiodarone before or during the study. The patients were given $100 \mathrm{mg}$ atenolol orally 4 hours before the study, to avoid the complication of variable adrenergic status, and diazepam $10 \mathrm{mg}$ orally an hour before the study. Measurements were made during

Table 1 Patient data and steady state intervals used in the study

\begin{tabular}{llll}
\hline Patient No & Sex & Age & $\begin{array}{l}\text { Steady state interval } \\
(\mathrm{ms})\end{array}$ \\
\hline 1 & M & 73 & 800 \\
2 & F & 72 & 800 \\
3 & M & 60 & 800 \\
4 & M & 61 & 800 \\
5 & M & 58 & 800 \\
6 & M & 38 & 600 \\
7 & M & 74 & 500 \\
8 & F & 56 & 600 \\
9 & F & 51 & 500 \\
10 & M & 58 & 500 and 700 \\
11 & M & 71 & 800 and 750 \\
12 & F & 57 & 500 and 800 \\
13 & & & \\
\hline
\end{tabular}

quiet breathing. The study was approved by the ethics committee of the participating hospitals. Informed consent was obtained from each patient before the study.

\section{MEASUREMENTS}

\section{Endocardial action potentials}

An action potential catheter as modified by Miller $e t$ $a l^{12}$ was used. The catheter was passed from the right femoral vein to the right ventricle and was allowed to touch the endocardial surface. A site on the septum towards the middle of the ventricle was chosen whenever possible to minimise variability between action potential duration and the QT interval. The potential difference between the two silversilver chloride electrodes of the catheter was measured with a DC amplifier and an oscillographic or electrostatic recorder. Recordings were made simultaneously on to a Racal instrumentation tape recorder. The duration of the action potentials (expressed as time during which the myocardial cells were depolarised by more than $70 \%$ ) was measured manually from all records at a fast paper speed. A standard electrocardiogram was monitored throughout the procedure. When we analysed the action potential duration we always checked the electrocardiographic signal to ensure that there were no spontaneous beats. QT interval was measured simultaneously from the standard electrocardiogram.

\section{Pacing}

The heart was stimulated by a right atrial (3 patients) or right ventricular (11 patients) bipolar pacing catheter introduced from the right femoral vein. If the atrioventricular node became refractory during attempted atrial pacing, ventricular pacing was substituted. Stimuli of $2 \mathrm{~ms}$ duration and twice threshold strength were produced from an isolated constant current source (Devices 2533) which was triggered by a programmable stimulator.

\section{Pacing protocols}

To determine the electrical restitution curves, the hearts were paced at a constant base cycle length (see table 1 for details) until action potential duration had reached a steady state. This was usually in 2-3 minutes. In the first five studies we used a basic pacing interval of $800 \mathrm{~ms}$ (table 1). This was chosen because it was expected to be comfortably shorter than the spontaneous $R-R$ interval of patients on $\beta$ adrenergic blockade. We then wished to check the effect of higher basic pacing frequency and used intervals of 500 or $600 \mathrm{~ms}$ (patients 6, 7, 9, and 10; table 1). We were also able to study two basic frequencies in individual patients (patients $8,11,12$, 
and 14; table 1). After each period of steady pacing at these intervals a test stimulus was applied after the refractory period of the last regularly paced response via the programmable stimulator. After each test stimulus, regular pacing at the basic cycle length was resumed for at least ten beats to allow recovery of the original steady state. Test pulse intervals were applied with either a progressively longer or shorter interval in sequence, in each patient. Action potential durations were measured at $70 \%$ repolarisation and plotted against test cycle length. For pooled data, the duration of the action potential in response to the test beats was expressed as a percentage of that of the steady state beats. When a frequency switch of steady state interval was made (patients $8,11,12$, and 14) the time taken for the response to reach a new steady state was carefully monitored.

\section{Results}

The monophasic action potential signals recorded with the electrode catheter were of good quality for determination of action potential duration (fig 1). As the interval preceding a test stimulus was extended, the duration of the action potential also increasedsee the electrical restitution curve (fig 2a). The results in the three patients in whom the protocol could be carried out by atrial pacing without atrioventricular conduction problems were similar to those in the 11 patients studied by right ventricular pacing. We have averaged the data for all those studies in which the steady state interval was $800 \mathrm{~ms}$ (fig $2 b$ ). This data does not include some patients in
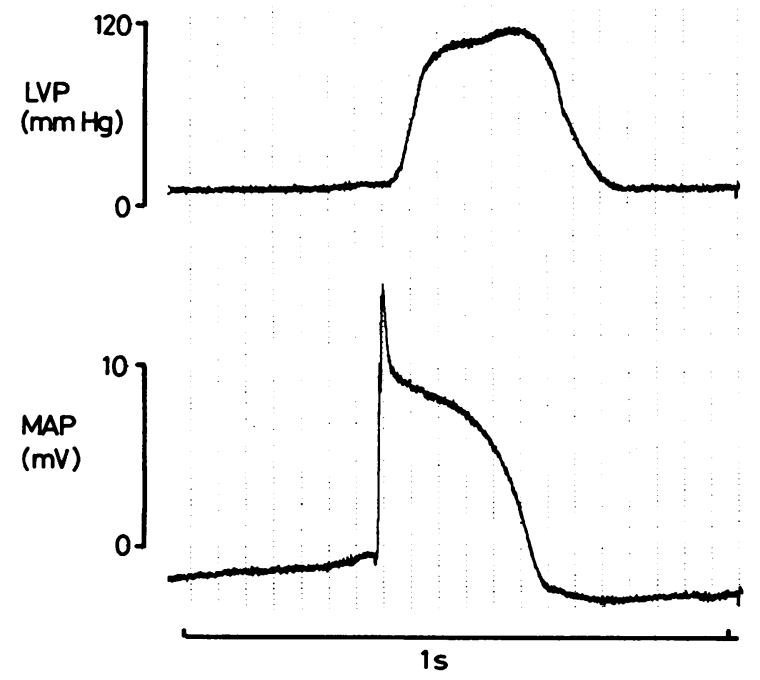

Fig 1 Recordings of typical monophasic action potential signal (MAP) from the human heart and left ventricular pressure (LVP). whom the spontaneous rate was too high to allow steady pacing at $800 \mathrm{~ms}$ intervals. The electrical res $=$ titution curves in these patients were similar, how $\overrightarrow{\overline{\bar{F}}}$ ever. In every patient there was a smooth increase it action potential duration with interval over the whole range studied. A transient decrease at inter. mediate intervals, as seen in two patients of Fran $\bar{\Phi}$ et al, ${ }^{6}$ was not seen in any of the present patients studied.

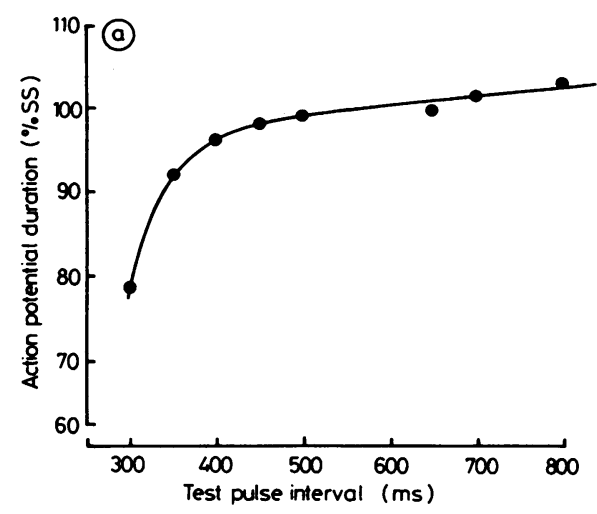

Fig 2 Electrical restitution curves in which action potential duration of the test beats as a percentage of steady state control action potential duration is plotted as a function of test pulse interval. (a) A typical individual example; (b) mean results (range) for all patients in whom the steady stat interval was $800 \mathrm{~ms}$.

In four patients we also studied the electrical res titution curve at a higher steady state frequency? This intervention was not possible in some patients because of the occurrence of angina or because it was not advisable to prolong the study. In the cases where results at two frequencies were obtained, the action potentials for all test pulse intervals weré shortened at the higher frequency; this resulted in downward shift of the electrical restitution curve (fi 3). Table 2 summarises the data from all patients.

Figure 4 illustrates the effects of a sudden switch in frequency of stimulation. On the first beat at the high frequency, the action potential was shortene\& to a degree that was consonant with the electrical restitution curve for the control (low) frequencye 
Table 2 Action potential duration measurements for the individual test pulse intervals used in the study

\begin{tabular}{|c|c|c|c|c|c|c|c|c|c|c|c|c|c|c|c|c|c|}
\hline \multirow[b]{2}{*}{ Pattent. No } & \multicolumn{17}{|c|}{ Interval } \\
\hline & 300 & 350 & 400 & 450 & 500 & 550 & 600 & 700 & 800 & 900 & 1000 & 1100 & 1200 & 1300 & 1400 & 1500 & 1600 \\
\hline 1 & - & 235 & 268 & 275 & 280 & 283 & 289 & 280 & 280 & 277 & 284 & 282 & - & - & - & - & - \\
\hline 2 & 256 & 287 & 301 & - & 292 & - & 299 & 300 & - & 302 & 312 & 310 & 320 & - & - & - & - \\
\hline 3 & - & - & - & 260 & 255 & 270 & 288 & 297 & 309 & 300 & 308 & 312 & 313 & 318 & - & - & - \\
\hline 4 & 199 & 246 & 248 & - & 242 & - & 253 & 265 & - & 256 & 259 & 254 & 267 & - & - & - & - \\
\hline 5 & 194 & - & 233 & - & 237 & - & 244 & 252 & - & 250 & 250 & 260 & - & - & - & - & - \\
\hline 6 & 209 & 242 & 255 & 258 & 259 & 262 & 263 & 266 & - & - & - & - & - & - & - & - & - \\
\hline 7 & - & - & 230 & 230 & - & 250 & 260 & 260 & 265 & 280 & 280 & 285 & 300 & 300 & 300 & - & - \\
\hline $8 a$ & - & - & 203 & 207 & - & 216 & 221 & 228 & 232 & 239 & 244 & 248 & 252 & 253 & 254 & 260 & 270 \\
\hline$b$ & - & - & 二 & 257 & 256 & 260 & 262 & 270 & 275 & 282 & 289 & 290 & 297 & 302 & 302 & 305 & 268 \\
\hline 9 & - & - & - & 192 & 200 & 202 & 206 & 218 & 222 & - & - & - & - & - & - & - & - \\
\hline 10 & 255 & 276 & 276 & - & 276 & - & 300 & - & - & - & - & - & - & - & - & - & - \\
\hline $1 \mathrm{la}$ & 206 & 218 & 218 & 222 & - & - & 210 & 206 & 210 & - & - & - & - & - & - & - & - \\
\hline b & 213 & 242 & 242 & 254 & 270 & - & - & 280 & 280 & 280 & 276 & - & - & - & - & - & - \\
\hline $12 \mathrm{a}$ & 200 & 203 & 207 & - & 213 & 213 & 218 & 217 & 223 & 223 & 228 & 228 & 237 & - & - & - & - \\
\hline b & 211 & 234 & 247 & 248 & - & - & - & 257 & - & - & 270 & - & - & - & - & - & - \\
\hline 13 & - & 265 & 290 & 293 & 305 & 300 & 303 & 310 & 321 & 333 & 325 & - & 350 & - & - & - & - \\
\hline $14 a$ & - & 235 & 237 & 237 & 235 & - & 237 & 250 & 258 & 269 & 295 & - & - & - & - & - & - \\
\hline b & - & - & - & 222 & 230 & - & 238 & 244 & 247 & 251 & 249 & 258 & 256 & - & - & - & - \\
\hline
\end{tabular}

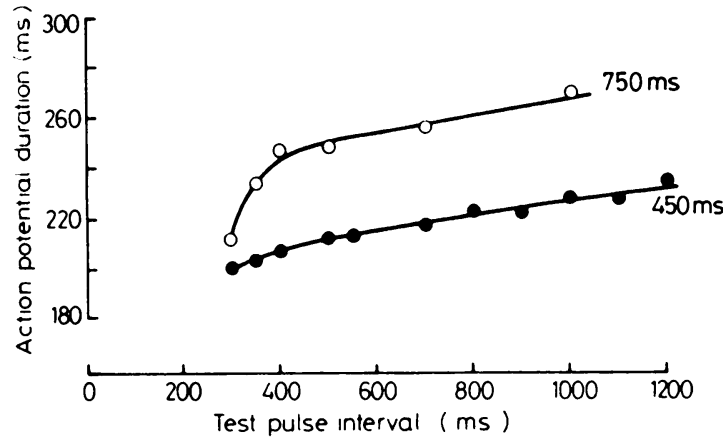

Fig 3 Two electrical restitution curces in one patient recorded at two different steady state intervals.
This was followed by a progressive further shortening for subsequent beats at the new (high) frequency. This shortening continued over several minutes (fig 4); even after three minutes there was still a trend to further action potential shortening with time. In all four cases the changes were qualitatively similar to those described for one patient by Franz et al. ${ }^{13}$

In those patients in whom adequate comparison could be made between action potential duration and simultaneous QT interval, the Spearman Rank correlation coefficient ranged from 0.80 to 0.88 . Most of the scatter contributing to the comparative analysis was derived from the QT measurements.

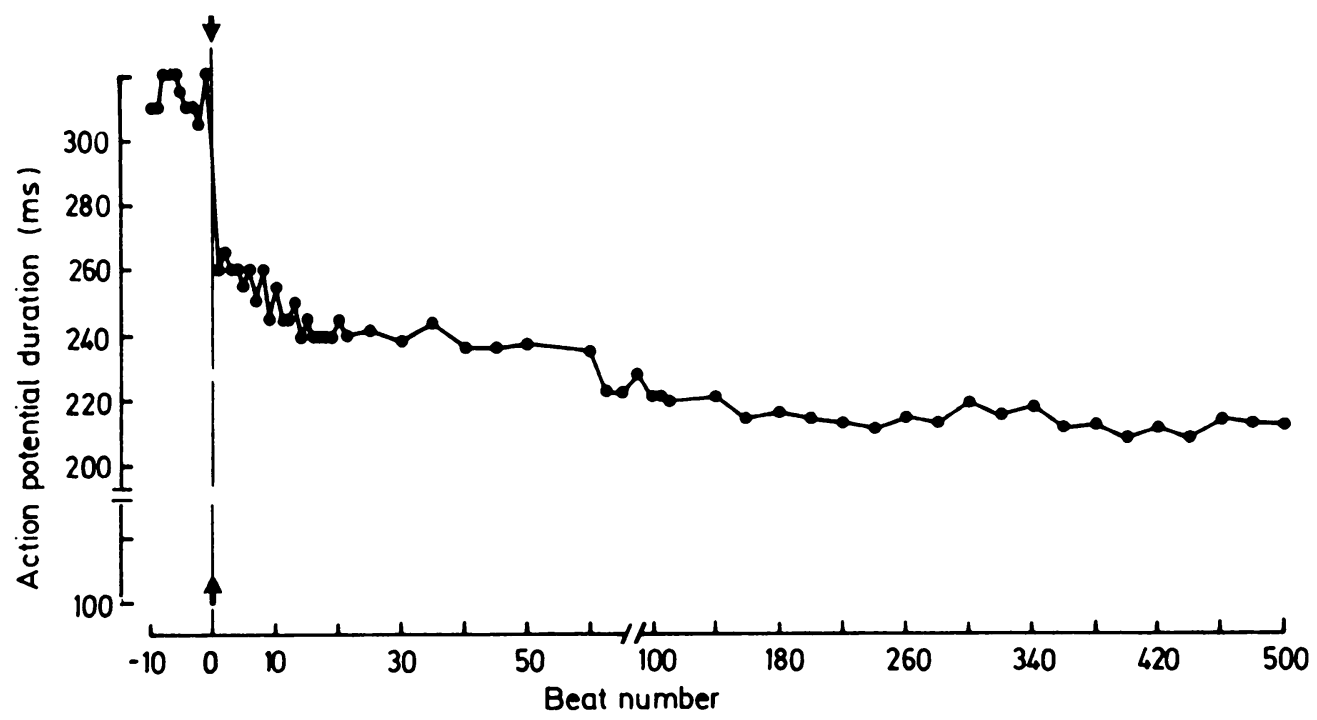

Fig 4 Beat to beat values for action potential duration after a sudden change of interval from 750 to $450 \mathrm{~ms}$. 


\section{Discussion}

Our results confirm that in the intact human heart the relation between action potential duration (and QT interval) and the R-R interval is similar to that of isolated cardiac muscle ${ }^{341415}$ and intact dog. ${ }^{5}$ This relation between action potential duration and test pulse interval is referred to as the electrical restitution curve. ${ }^{45}$

A previous report suggests that the electrical restitution curve of man was of a different form, namely a biphasic relation ${ }^{6}$ similar to that of the rabbit. ${ }^{16}$ There are species differences in the shape of electrical restitution curves. The shapes of the electrical restitution curves reported for two of the five patients by Franz $e t a l^{6}$ resemble those of the rabbit, in that action potential duration at intervals of $300-400 \mathrm{~ms}$ was prolonged. Action potential duration then declined again at frequencies between 400 and $500 \mathrm{~ms}$, before increasing again with longer intervals. We did not observe such changes in any of our 14 patients. In the study of Franz et al the prolonged action potentials at short preceding intervals were accompanied by changes in the shape of the action potential waveform. ${ }^{6} \mathrm{We}$ are of the opinion that monophasic signals do not give reliable information on action potential shape but only of action potential duration. ${ }^{17} \mathrm{We}$ would therefore place more reliance on the present measurements in a considerably larger group of patients, and we conclude that the human electrical restitution curve is very similar to that of the cat ${ }^{4}$ and of the dog. ${ }^{5}$

The electrical restitution curve determines the action potential duration and QT interval of only the first excitation after the first short $R-R$ interval. If short $R-R$ intervals are maintained by a continuation of pacing at a high frequency there is a further progressive shortening of the action potential duration and QT interval over a period of several minutes. This was reported for the QT interval of man by Attwell et $a l^{7}$ and for the action potential duration of one person. ${ }^{13}$ If the electrical restitution curve is redefined after such a period, it is found to show shorter action potential duration at all $R-R$ intervals. This downward shift of the electrical restitution curve when the steady state frequency is higher (fig 3 ) is very similar to the findings in the dog. ${ }^{5}$ The changes in action potential duration with a maintained frequency increase after a sudden switch (fig 4) are also very similar to findings in the dog for both Purkinje fibres ${ }^{18}$ and endomyocardial recordings in the intact animal. ${ }^{8}$

It would seem reasonable therefore to assume that the changes we see are due to mechanisms that apply generally in higher animals. The immediate action potential shortening on the first beat is a shift along the restitution curve for the low steady state fre quency. The restitution curve depends on changes: in factors affecting recovery of the cell membran $\overrightarrow{\vec{F}}$ after a depolarisation. This is thought to be due part to recovery from inactivation of the slow inwarf calcium channels. ${ }^{18}$ There is also decay of outwar每 potassium currents during the polarised period (oD electrical diastolic interval) ${ }^{6}$ which may be inter rupted by shorter intervals. The mechanisms under - ? lying the second, slower phase of further action potential shortening during a period of high fre $\overrightarrow{=}$ quency pacing (fig 4) were examined in some detaiw in the intact dog by Drake et al. ${ }^{8}$ The slow time course of change in action potential duration after aner increase in stimulation frequency suggested that these changes are caused by the accumulation of ariw ion or metabolite, or possibly by changes in activity of the electrogenic $\mathrm{Na}^{+}-\mathrm{K}^{+}$pump ${ }^{18}$; such mech? anisms appear to act via an increase in outward back $\vec{\imath}$ ground current. ${ }^{18}$ Whatever mechanism is responsible for this change, it is clearly maintaine $\bar{\epsilon}$ for at least $1400 \mathrm{~ms}$.

The results of our study are relevant to argument $\overrightarrow{80}$ concerned with the corrections to be made to QT interval to allow for heart rate. ${ }^{19-21}$ Thus from oug results and from those using QT intervals ${ }^{7}$ it mus? be concluded that the relation between heart rate and QT interval is not constant. For instantaneous differences in interval between beats, QT intervad must be governed by the electrical restitution curve (fig 1). For more prolonged periods, the relation wils also be affected by the slower changes in action potential duration, which are time dependent (fig 4 ) We have confirmed that after one beat at a different interval, it takes up to 10 beats for a steady state to b regained. When there is a switch from one sustaine period at a given frequency to a new frequency, it takes at least three minutes for a new steady state to be reached.

Our study does not explore the additional influence of the sympathetic nervous system upon action potential duration and QT interval, becaus? our patients were deliberately subjected to $\beta$ adre nergic blockade by atenolol. An increase in sym $N$ pathetic activity in the absence of such blockades causes a further shortening of action potentiap duration at a given fixed heart rate ${ }^{22}$; this effect is valuable in producing an increase in heart rate witk exercise in appropriately programmed pace? makers. $^{22}$ Any correction of QT interval for hear\$ rate that ignores changes that might have occurred during the preceding five minutes must therefore bo unreliable. Such dubious corrections are sometimes used in human clinical pharmacological studies-fof example class III antiarrhythmic agents. From ou? study we conclude that the use of rate corrected $Q$ 
interval is not valid. We suggest that such studies should be carried out at constant heart rate achieved by pacing for at least three minutes.

\section{References}

1 Pidgeon J, Lab MJ, Seed WA, Elzinga G, Papadoyannis D, Noble MIM. The contractile state of cat and dog heart in relation to stimulation frequency. Circ Res 1980;47:559-67.

2 Pidgeon J, Miller GAH, Noble MIM, Papadoyannis $D$, Seed WA. The relationship between the strength of the human heart beat and the interval between beats. Circulation 1982;65:1404-10.

3 Bass BG. Restitution of the action potential in cat papillary muscle. Am F Physiol 1975;228:1717-24.

4 Boyett MR, Jewell BR. A study of the factors responsible for rate-dependent shortening of the action potential in mammalian ventricular muscles. f Physiol (Lond) 1978;285:359-80.

5 Elzinga G, Lab MJ, Noble MIM, et al. The action potential duration and contractile response of the intact heart related to the preceding interval and the preceding beat in the dog and cat. $F$ Physiol (Lond) 1981;313:481-500.

6 Franz MR, Schaefer J, Schoettler M, Seed WA, Noble MIM. Electrical and mechanical restitution of the human heart at different rates of stimulation. Circ Res 1983;53:815-22.

7 Attwell D, Cohen I, Eisner DA. The effect of heart rate on the action potential of guinea-pig and human ventricular muscle. F Physiol (Lond) 1981;313:439-61.

8 Drake AJ, Noble MIM, Schouten V, Seed WA, ter Keurs HEDJ Wohlfart B. Is action potential of the intact dog heart related to contractility or stimulus rate? f Physiol (Lond) 1982;331:499-510.

9 Bazett HC. An analysis of the time relations of the electrocardiograms. Heart 1920;7:353-69.

10 Shipley RA, Hallaran WR. The four-lead electrocardiogram in two hundred normal men and women. Am Heart f 1936;11:325-45.
11 Lepeschkin E. Modern electrocardiography, vol. 1. Baltimore: Williams and Wilkins, 1951.

12 Miller GAH, Noble MIM, Papadoyannis D, Pidgeon J, Seed WA. A catheter-tip method for recording monophasic action potentials from the canine or human endomyocardium. F Physiol (Lond) 1980; 305:7-8P.

13 Franz M, Schoettler M, Schaefer J, Seed WA. Simultaneous recording of monophasic action potentials and contractile force from the human heart. Klin Wochenschr 1980;58:1357-9.

14 Hauswirth O, Noble D, Tsien RW. The dependence of plateau currents in cardiac Purkinje fibres on the interval between action potentials. F Physiol (Lond) 1972;222:27-9.

15 Gettes LS, Reuter H. Slow recovery from inactivation of inward currents in mammalian myocardial fibres. f Physiol (Lond) 1974;240:703-24.

16 Wohlfart B. Relationships between peak force, action potential duration and stimulus interval in rabbit myocardium. Acta Physiol Scand 1979;106:395-409.

17 Hoffman BF, Cranefield PF, Lepeschkin E, Surawicz B, Herrlich HC. Comparison of cardiac monophasic action potentials recorded by intracellular and suction electrodes. Am F Physiol 1959;196:1297-301.

18 Boyett MR, Fedida D. Changes in the electrical activity of dog cardiac purkinje fibres at high heart rates. f Physiol (Lond) 1984;350:361-91.

19 Kelman AW, Whiting B, Sumner DJ. Correction equation for ECG time intervals. Br $\mathcal{F}$ Clin Pharmacol 1984;18:113-4.

20 Staniforth DH. The QT interval and cycle length: the influence of atropine, hyoscine and exercise. $\mathrm{Br} \mathcal{F}$ Clin Pharmacol 1983;16:615-21.

21 Evans SJW, Kelman AW, Whiting B, Sumner DJ, Staniforth DH. Correction equation for ECG time intervals; correction equation for QT interval [Correspondence]. Br $\mathcal{F}$ Clin Pharmacol 1985;19: 860-2.

22 Rickards AF, Norman J. Relation between QT interval and heart rate: new design of physiologically adaptive cardiac pacemaker. Br Heart $\mathcal{f} 1981$;45:56-61. 